\title{
ILCEA
}

Revue de l'Institut des langues et cultures

d'Europe, Amérique, Afrique, Asie et Australie

$12 \mid 2010$

La FASP : dix ans après...

\section{El thriller académico en la literatura española: $L a$ Cátedra de Javier Piqueras de Noriega}

Le thriller universitaire dans la littérature espagnole: La Cátedra de Javier

Piqueras de Noriega

The academic thriller in Spanish literature: La Cátedra by Javier Piqueras de

Noriega

\section{Cécile Petit}

\section{OpenEdition}

\section{Journals}

Edición electrónica

URL: http://journals.openedition.org/ilcea/580

DOI: 10.4000/ilcea.580

ISSN: 2101-0609

Editor

UGA Éditions/Université Grenoble Alpes

Edición impresa

ISBN: 978-2-84310-180-9

ISSN: 1639-6073

Referencia electrónica

Cécile Petit, «El thriller académico en la literatura española: La Cátedra de Javier Piqueras de Noriega », ILCEA [En línea], 12 | 2010, Publicado el 28 enero 2014, consultado el 30 abril 2019. URL : http:// journals.openedition.org/ilcea/580 ; DOI : 10.4000/ilcea.580

Este documento fue generado automáticamente el 30 abril 2019.

(C) ILCEA 


\title{
El thriller académico en la literatura española: La Cátedra de Javier Piqueras de Noriega
}

\author{
Le thriller universitaire dans la littérature espagnole : La Cátedra de Javier \\ Piqueras de Noriega \\ The academic thriller in Spanish literature: La Cátedra by Javier Piqueras de \\ Noriega
}

Cécile Petit

Javier Piqueras de Noriega, universitario y escritor español, publicó en 2003 su primera novela, titulada La Cátedra. ${ }^{1}$ Este libro, seguido dos años más tarde por la publicación de $E l$ Congreso $^{2}$ con el que comparte la misma índole, inaugura en España el nacimiento de un género hasta ahora descartado por los autores españoles, y con todo frecuentemente desarrollado por autores anglófonos.

En efecto, en varios artículos, el anglicista francés Michel Petit se refiere al surgimiento en el ámbito literario anglófono de un nuevo género, que nombra « Ficción de substrato profesional $»,{ }^{3}$ constituido por un conjunto de ficciones que ofrecen aspectos comunes: son novelas caracterizadas por un argumento que se desarrolla en un sector profesional preciso, por personajes que forman parte de ese mismo medio, y escritas por autores que pertenecen, han pertenecido, o así lo pretenden, a este dominio laboral. ${ }^{4}$ Estas ficciones ancladas en un medio laboral específico se presentan casi siempre como thrillers. Son thrillers profesionales, o thrillers especializados, ${ }^{5}$ entre cuyas variantes está el thriller académico, mezcla del campus novel ${ }^{6}$ que ya existía antes y del thriller profesional que apareció hace poco.

Con su novela, Javier Piqueras se acerca al campus novel, y más específicamente al thriller universitario, de tal modo que por primera vez un autor español se apropia con mucho talento de un género que parecía ser un coto reservado a escritores anglófonos.7 Destacaremos aquí las características externas e internas, o sea peritextuales y narrativas, que permiten a La Cátedra presentarse como un excelente thriller académico. 


\section{La Cátedra y las características peritextuales del thriller académico}

Michel Petit y Jean-Pierre Charpy subrayan que varios elementos peritextuales constituyen los rasgos definitorios del thriller especializado y permiten identificar en seguida si un libro pertenece a este género. Primero el peritexto pone de relieve que la novela es un thriller a través de la palabra «thriller » que suele figurar de manera aislada en la tapa, en la contratapa o en las solapas, que viene incluida en la biografía del autor o en la presentación de la obra que a menudo se imprimen en estas partes del libro, o mediante el resumen de contratapa que resalta el suspense inherente al argumento. ${ }^{8}$ Así sucede en la novela de Javier Piqueras de Noriega. En la solapa, un breve párrafo define La Cátedra como un "thriller ", alude a los « entresijos " representados en el libro mientras que en la contratapa una presentación de la novela sobreentiende que La Cátedra forma parte de este género puesto que se señala que es una "interesante novela de intriga ", que presenta «episodios de ambición, corrupción y violencia». Por otro lado, la contratapa incluye un resumen del argumento lleno de referencias a ingredientes de este género: problemas y sucesos raros vividos por varios personajes, una denuncia, delitos, interrogatorios, investigaciones realizadas por diferentes personajes. Estos elementos hacen que el lector entienda de antemano que se trata de un thriller. Además, algunos elementos gráficos lo corroboran. De manera general y, en particular, en el caso de los thrillers, el diseño de la tapa atrae e informa al lector indicándole visualmente la definición genérica y temática del libro. ${ }^{9}$ Así, en la cubierta de La Cátedra, la foto de una cama con una cinta atada a la barra de la cabecera hace pensar en escenas de violencia típicas del thriller.$^{10} \mathrm{El}$ índice o más especialmente varios títulos de capítulos anunciados en el sumario de La Cátedra evocan también la pertenencia de la novela al thriller, porque las palabras «enemigos ", " paranoia », « acertijos », « ruidos y gemidos $»^{11}$ aluden a situaciones o ingredientes de este género. Con todo, el peritexto no sólo subraya que se trata de un thriller sino también de un thriller especializado.

Mediante elementos peritextuales, se precisa el género al que pertenece la novela, se destaca el subgrupo genérico del que forma parte: es un thriller especializado, un thriller relacionado con un sector profesional específico. ${ }^{12}$ La referencia al dominio laboral y, por tanto, al género del que depende La Cátedra se lleva a cabo especialmente gracias al título del libro. El título se refiere directamente a un elemento propio del sector universitario y anuncia de antemano al lector que la trama se ubica en el ámbito académico. Los elementos gráficos permiten llamar la atención del público e informarlo de un simple vistazo que el relato se relaciona con este medio ya que en la tapa y la contratapa figuran las fotos de un anfiteatro y de estanterías con libros. Además, el resumen del argumento impreso en la contraportada alude al mundo universitario mencionando desde el principio que los personajes de la novela son entre otros un profesor, candidatos a una cátedra y una estudiante, evocando actividades propias de este medio laboral como la participación en tribunales de oposición. La presentación que se hace del libro en la contraportada y en la solapa también sobreentiende su vínculo con el sector académico: La Cátedra es una novela " perfectamente ambientada en el mundo universitario », " una obra que refleja (...) el amplio conocimiento que tiene Piqueras del mundo universitario ». El índice contiene títulos de capítulos que se refieren a situaciones típicas de este medio 
laboral, evocadas por las palabras «comisión » y «comunicación », ${ }^{13}$ y corrobora la ubicación de la trama en dicho ámbito.

Por otro lado, la referencia al dominio profesional se realiza gracias a la presencia de datos biográficos. El peritexto suele proporcionar informaciones sobre el autor que permiten mostrar su íntima relación con el medio laboral tratado en la novela: a menudo se mencionan su profesión, el lugar donde trabaja o trabajó, sus años de experiencia en este oficio, los estudios que cursó, los diplomas que obtuvo, elementos curriculares que se vinculan casi siempre con el sector profesional considerado en la ficción. ${ }^{14} \mathrm{El}$ hecho de que un escritor sea o haya sido un actor del sector sobre el que escribe pone de relieve su conocimiento profundo del medio al que se refiere, lo convierte en especialista de este dominio y garantiza el valor realista del substrato profesional presente en el libro. ${ }^{15} \mathrm{El}$ peritexto de La Cátedra comparte estas características. En la solapa, varias informaciones biográficas presentadas como si se tratara prácticamente de un historial, subrayan la relación de Javier Piqueras de Noriega con el sector universitario representado. Se nos indica su formación, es decir, los estudios que hizo y el título que consiguió: «estudió Ciencias Físicas en la Universidad Complutense, donde realizó un doctorado ». Luego una serie de elementos relativos al currículum profesional del autor informan al lector de que es "catedrático", esto es "profesor e investigador», especializado en "Física de Materiales ", que trabaja y trabajó en varias universidades y centros de investigación de diversos países europeos. Estos datos presentan a Javier Piqueras como especialista de la materia evocada en el libro. Además, algunos elementos gráficos suelen corroborar el hecho de que el autor de este tipo de novelas trabaje o haya trabajado en el sector profesional al que se refiere, pues su fotografía -sacada en su lugar de trabajo y con ropa de trabajo- está casi siempre en la contratapa o en la solapa. ${ }^{16}$ De hecho en la solapa de $L a$ Cátedra vemos la foto del escritor delante de una estantería con libros, a lo mejor tomada donde trabaja o por lo menos de tal modo que el lector pueda pensarlo. Ese elemento visual hace que el escritor presentado como un profesional del medio que describe, aparezca en carne y hueso, lo cual da aún más crédito al substrato profesional del argumento. Michel Petit señala que a veces esos datos biográficos no son ciertos, pero son necesarios para indicar el género de la novela y dar valor de autenticidad a estos thrillers especializados. ${ }^{17}$ No es el caso de La Cátedra, ya que varios documentos demuestran que efectivamente Javier Piqueras realiza la actividad profesional indicada. Ello lo corroboran las informaciones encontradas en el sitio internet de la editorial ${ }^{18} \mathrm{y}$, sobre todo, de la Universidad Complutense de Madrid a partir del cual se accede a las páginas de las Facultades, los Departamentos y los miembros de la Complutense, entre las cuales está la página del Catedrático y Director del Departamento de Física de Materiales: Javier Piqueras. ${ }^{19}$

Mientras que esos elementos peritextuales, que remiten a la vez a la intervención del editor y del escritor, ${ }^{20}$ subrayan que el autor conoce muy bien el sector académico, e incluso permiten afirmar que su conocimiento del medio se «refleja » en la obra, otro componente del peritexto parece hacer hincapié en la falta de correspondencia entre el contenido de la novela y la realidad. Se trata de una advertencia escrita por el autor:

Los hechos, personajes, instituciones y empresas que aparecen en esta novela e incluso los fenómenos físicos que se describen en ella son ficticios. Cualquier posible parecido con la realidad es una coincidencia involuntaria. ${ }^{21}$

El autor da la impresión de negar la existencia de una relación entre su ficción y la realidad, de poner en tela de juicio la presunta autenticidad de su relato. Pero rechazar la idea de que la ficción pueda ser un reflejo de la realidad también permite sugerir esa 
eventualidad. Este tipo de advertencia, frecuentemente utilizada en las series o en las películas policíacas de las que Javier Piqueras reconoce cierta influencia en su escritura, tiene generalmente como meta proteger al autor en caso de que alguien o alguna entidad o institución presente una denuncia por verse representada o criticada en una obra. La presencia de este aviso procede precisamente de la similitud entre ficción y realidad. Por tanto, la advertencia afirma todo lo contrario de lo que pretende declarar: sí, el relato tiene semejanzas con lo real. Es más, esta advertencia puede ser irónica o resultar de la intención satírica del escritor. En este caso, consiste en dar a entender lo contrario de lo que expresa, para insistir en el carácter realista de la novela o para recordar que el objetivo de la deformación satírica de la realidad es, al final, representarla y criticarla. De esta manera, la observación preliminar del escritor refuerza la probabilidad de que haya una vinculación entre lo ficticio y lo real e indica su voluntad de proponerle al lector una representación del mundo laboral universitario. Por consiguiente, este aspecto del peritexto, junto con los demás elementos peritextuales que evocamos, anuncia el contenido del libro: La Cátedra es más que un campus novel, es un thriller académico.

\section{La Cátedra y las características narrativas del thriller académico: de la representación de la realidad profesional al thriller}

Según Michel Petit, el hecho de que una novela sea un thriller especializado significa que la caracteriza antes que nada « la presencia permanente e imprescindible, en el contenido narrativo y en la escritura misma, de la representación predominante de una realidad profesional que llega a ser, precisamente por eso, el verdadero substrato de la ficción $»^{22}$ Ello quiere decir que para él un thriller especializado es un thriller estrechamente relacionado con un medio profesional que, de hecho, constituye la razón de ser de la novela, mientras que el género constituido por el thriller se utiliza antes que nada como pretexto para atraer al gran público. ${ }^{23}$ Este no es el caso de La Cátedra. En efecto la novela de Piqueras ofrece una representación realista del sector laboral universitario pero, además, varios aspectos profesionales representados son ingredientes inherentes del thriller. Dicho de otro modo el thriller surge precisamente del substrato profesional.

\section{La representación de la realidad profesional}

Al desarrollarse el argumento de estos thrillers especializados en un medio laboral específico, se citan o describen sitios, objetos, personas, actividades que lo caracterizan..$^{24}$ En La Cátedra, el narrador se refiere a varias universidades, ficticias, en particular a cuatro que son la Universidad de Madrid, la Universidad Pública Miguel de Cervantes, la Universidad Científica de Cataluña, el Instituto Politécnico de Boston, lo cual favorece la multiplicación de alusiones a aspectos típicos del universo académico. Se aprecian muchas referencias a lugares que son propios del espacio universitario y que corresponden a los diferentes sitios que constituyen una universidad. Se alude varias veces a la apariencia general de las universidades, lo que nos permite distinguir dos tipos de universidades. La Universidad de Madrid y la Universidad Científica de Cataluña son universidades más antiguas, construidas según modelos españoles, recientemente sometidos a remodelación, que contrastan con la Universidad Pública Miguel de Cervantes y el Instituto Politécnico de Boston, las cuales constituyen universidades 
modernas edificadas a partir del modelo anglosajón de campus. Se mencionan zonas específicas de las universidades típicamente españolas, los Colegios Mayores, y, sobre todo, se evocan frecuentes obras, que corresponden a la imprescindible adaptación de los locales de las viejas universidades a las necesidades actuales. No sólo los edificios fueron remodelados para crear centros de investigación, sino que fueron ampliados para adecuar su superficie al aumento del número de estudiantes debido a la democratización de los estudios superiores que se produjo en España durante las últimas décadas. Asimismo, estos edificios se ajustaron a las normas de seguridad. ${ }^{25}$ Este último aspecto refleja la índole vetusta de esas dos universidades poco comparables con el moderno campus Miguel de Cervantes que dispone en particular de un edificio calificado como " espectacular», "elegante», "funcional», semejante a "la sede central de alguna empresa importante $»{ }^{26}$ De la misma manera, las partes exteriores de esas universidades presentan pocas similitudes, pues con sus jardines la Universidad de Madrid tiene poco que ver con la Miguel de Cervantes y sus amplios espacios exteriores con aparcamientos, su «gran extensión de césped (...) que producía una impresión más británica que madrileña $"{ }^{27}$ y aún tiene menos en común con el Instituto Politécnico de Boston pues, más que concentrar en un mismo sitio departamentos, lugares de docencia y de investigación, servicios administrativos y bibliotecas como la Miguel de Cervantes, el campus del Politécnico de Boston es una verdadera ciudad miniatura. ${ }^{28}$

A pesar de estas diferencias arquitectónicas exteriores, las cuatro universidades ofrecen los mismos servicios, con excepción de los alojamientos que sólo existen en el campus norteamericano. El lector desconocedor de este medio entiende que consta de diferentes facultades y departamentos, aunque sólo se citan la Facultad de Física de la Universidad de Madrid, la Facultad de Ciencias de la Universidad Científica de Cataluña y el Departamento de Ciencias Aplicadas de la Universidad Pública Miguel de Cervantes. ${ }^{29} \mathrm{El}$ lector puede familiarizarse con algunos de sus servicios administrativos, en especial con las Secretarías y la Dirección. ${ }^{30}$ También se evocan o describen espacios dedicados a profesores, investigadores y doctorandos. Hay referencias a sus despachos, ${ }^{31}$ algunas subrayan en particular la diferencia que puede haber entre docentes e investigadores reconocidos por su trabajo y otros que no. Así pues, mientras los despachos del personal de la Universidad Científica de Cataluña están localizados en la parte moderna, el despacho de una profesora se halla aislado de los de los demás y deja una impresión negativa pues se caracteriza por el desorden, lo viejo y el estancamiento..$^{32}$ Aparte de los despachos, se alude a diferentes salas destinadas a la docencia, al estudio o a la investigación, como las aulas, entre las cuales destacan las aulas donde se realizan « las clases de prácticas de laboratorio ", la sala de informática, de la cual hasta se nos indica la hora de cierre, las bibliotecas, los laboratorios, los locales técnicos o talleres donde trabajan los técnicos de los laboratorios, así como la Sala de Actos, lugar que se relaciona directamente con el tema principal del libro pues allí se realizan, entre otras cosas, las entrevistas de oposición para cátedra. ${ }^{33}$

La referencia a esos lugares implica también la representación de objetos o utensilios utilizados en este sector profesional. Como lo subraya Michel Petit, la presencia de objetos no tiene como meta principal crear cierto realismo sino informar sobre el medio laboral que constituye el tema de La Cátedra. Mientras el personal administrativo utiliza los mismos instrumentos que en cualquier tipo de empresa, o sea ordenadores, fotocopiadora, carpetas, lápices o grapadoras ${ }^{34}$ el material utilizado por el personal docente proporciona más informaciones sobre lo específico del sector laboral 
representado. Por un lado, se representan las cosas que suelen utilizarse tradicionalmente. Los profesores tienen un « casillero », una " carpeta con los apuntes de clase», archivadores, ficheros con fichas de estudiantes matriculados en la asignatura, listas de alumnos que se examinan. ${ }^{35}$ Por supuesto, la pizarra forma parte de los instrumentos de trabajo clásicos para la enseñanza; sin embargo, en la novela se trata de pizarras actuales, o sea blancas en las que se escribe con rotuladores de colores. ${ }^{36}$ También se alude al uso de transparencias que utilizadas con un proyector, permiten mostrar documentos y gráficos. ${ }^{37}$ Pero, por otro lado, se representan objetos mucho más modernos, como ordenadores para navegar por internet y comunicar por mensajería electrónica, realizar presentaciones y proyectar la pantalla del ordenador en vez de usar el proyector de transparencias. ${ }^{38}$ Con todo, el instrumento principal del profesor sigue siendo su voz. ${ }^{39}$ Además se representan objetos y materiales empleados por el personal investigador, es decir, equipamiento científico como aparatos de medidas electrónicas o equipo de " resonancia de superficies $"{ }^{40}$ dándonos al mismo tiempo detalles a propósito del precio que cuesta reparar este tipo de material ${ }^{41} \mathrm{o}$ explicándonos el contenido de un « armario modular »:

Se veía un aparato formado por numerosos tubos relucientes de acero inoxidable, varios de los cuales estaban acoplados a una cámara, también metálica y de un metro de altura. En la cámara, unas ventanas como pequeños ojos de buey de un barco, permitían observar su interior. Del aparato salía una maraña de cables que en su mayor parte estaban conectados a los módulos en los que trabajaba Eva y en los que una serie de luces piloto y numerosos rojos y amarillos en pequeñas pantallas cambiaban continuamente. También había varios ordenadores conectados a todo el sistema. ${ }^{42}$

Se especifican las condiciones de seguridad que hay que cumplir para utilizar este tipo de material, son imprescindibles « instalaciones eléctricas modernas ", " circuitos cerrados de refrigeración ", " conducción de gases ».43 También hay referencias a materiales complementarios como cables, herramientas, soldadores, ${ }^{44}$ que sirven para arreglar los equipos y que suelen formar parte de los objetos empleados por los técnicos, encargados de preparar y reparar los aparatos utilizados en los laboratorios. Los locales técnicos están repletos de este tipo de material, el lector puede enterarse de ello a través de la descripción de un taller de electrónica:

El taller era una habitación casi cuadrada de unos tres metros de lado en la que prácticamente no había superficie libre. En las estanterías se amontonaban aparatos eléctricos, monitores de ordenador, libros de instrucciones y catálogos. Varios armarios metálicos colgados en la pared, con las puertas abiertas, contenían herramientas y pequeños cajones con tornillos y componentes electrónicos. En una gran mesa de trabajo estaban distribuidos un par de ordenadores sin carcasa y algunos aparatos medio desmontados al lado de manojos de cables y algunas herramientas y soldadores. ${ }^{45}$

Por otro lado, al suceder las acciones de la novela en diferentes sitios que forman el paisaje universitario, la narración incluye representaciones de personas que trabajan o acuden allí. Aparte de los estudiantes, que pueden ser doctorandos, doctorandos becarios y estudiantes de primer ciclo, ${ }^{46}$ se evoca el personal administrativo en especial las secretarias de departamento, así como el personal de dirección como el Rector de la Universidad y los jefes de departamentos o facultades. ${ }^{47}$ También se menciona el personal técnico, lo que permite aludir a la existencia de diferentes estatutos laborales para la gente que trabaja en las universidades, a la precariedad de ciertos puestos de trabajo y a 
la incidencia de esa situación precaria en la posibilidad que tiene el trabajador de expresar su opinión:

No quiero meterme en líos. Yo estoy aquí con un contrato que me renuevan todos

los años y espero que me saquen la plaza a concurso para quedarme fijo. ${ }^{48}$

Además, se describe detenidamente el personal docente e investigador, indicando que existen diferentes estatutos en este cuerpo profesional, unos siendo catedráticos y otros aspirantes a tal puesto. ${ }^{49}$ En cuanto a su ropa, varios universitarios presentan una apariencia poco cuidadosa, anticuada, de mal gusto e incluso ridícula, ${ }^{50}$ que traduce la escasa importancia que dan a sus vestidos. Sin embargo, las malas condiciones financieras que suelen caracterizarlos explican al mismo tiempo que ciertos lleven vestidos desgastados o pasados de moda. ${ }^{51}$ La evocación de los universitarios implica la descripción de sus relaciones humanas, del ambiente peculiar que reina entre ellos, de las relaciones conflictivas que hay entre colegas:

Distaba mucho de ser la más popular entre el profesorado, especialmente entre sus compañeros de departamento. (...) Su última investigación de la que se tuvieran noticias era su tesis doctoral, que había presentado unos treinta años antes (...). La mayoría de los profesores más jóvenes realizaban una investigación activa con contactos internacionales, publicaciones y congresos y no estaban dispuestos a que una nulidad científica como Nuria les mirara por encima del hombro, ni mucho menos les diera instrucciones. Como reacción Nuria empezó a descalificar, siempre que tenía oportunidad, a los profesores que realizaban investigación científica ya que, según ella, descuidaban la docencia, que era para lo que estaban allí. Eso no aumentó su popularidad entre sus compañeros, que pensaban que además de investigar daban las clases mucho mejor que Nuria. ${ }^{52}$

Se sugiere aquí que los conflictos nacen de la incompetencia de ciertas personas que por amargura menosprecian a los compañeros que sí demuestran dinamismo y competencia. Pero es más: se señala la posibilidad de conseguir puestos o privilegios no por la calidad de sus investigaciones o por las capacidades manifestadas, sino por contactos y enchufe. ${ }^{53}$ Aparte de este clima relacional negativo entre universitarios, un clima semejante puede existir entre ciertos profesores y estudiantes, no sólo por los problemas planteados por dicha incompetencia, que implica cursos mal impartidos e investigaciones mal dirigidas, ${ }^{54}$ sino también por el poder excesivo que los docentes ejercen en ellos. Ciertos profesores se presentan como personas todopoderosas que por su influencia pueden hacer que un estudiante deje la carrera, no obtenga su diploma aunque cambie de universidad o no adquiera puesto, y que, por tanto, determinan su futuro: ${ }^{55}$

No tenéis ni idea de lo vengativo que es el jefe. Se cree que todos sus colaboradores tienen que opinar lo mismo que él, y tener los mismos amigos y enemigos. Algunas cosas que le he oído a veces te dejan alucinada. Hace tiempo estuve pensando pedir el traslado cuando lo de Jorge Marín. (...) Empezó a hacer la tesis y lo tuvo que dejar. Yo lo vi venir, por los comentarios que hacía el jefe cuando se enteró de que Jorge tomaba café con los del Departamento de Informática, que sabes que están peleados con Cañamero. Luego, todo eran pegas con el trabajo del Jorge, todo estaba mal. Parece que el jefe le hacía trabajar para otras cosas y Jorge no avanzaba con su tesis. Estaba desesperado y, cuando por fin decidió marcharse, Cañamero le echó una bronca y le dijo que se ocuparía de que no hiciera nunca su tesis en ninguna universidad española. (...) Te podría contar más historias pero prefiero no desmoralizarte. ${ }^{56}$

Referirse a personajes que son universitarios implica también representar sus actos profesionales. Michel Petit considera que la descripción precisa o técnica de actos profesionales no consiste en dar un aspecto realista al relato, sino en facilitar 
informaciones sobre el sector profesional tratado en la novela..$^{57}$ Para él, un thriller especializado es especializado antes de ser un thriller, es decir, que el suspenso y el argumento son antes que nada soportes narrativos para informar sobre un medio laboral. La Cátedra no encaja con esta idea. La representación de ciertas actividades permite proporcionar datos sobre el dominio académico, pero también influye en el desarrollo del argumento y es necesaria para la progresión de la historia. Veremos más adelante que, efectivamente, estas tareas profesionales forman parte integrante de la novela pues son aspectos específicos del mundo universitario e ingredientes imprescindibles del thriller. Primero una serie de elementos proporciona al lector las diferentes ocupaciones que forman parte del trabajo de los universitarios, sin que se dejen de lado su posible carácter fastidioso, los problemas de funcionamiento, la falta de ánimo del personal, que puede relacionarse con factores personales o con condiciones de trabajo poco satisfactorias. ${ }^{58}$

Para los lectores, la actividad que los define es impartir clases. Se evoca esta parte del trabajo ${ }^{59}$ así como aspectos relacionados con ella. Por ejemplo, hay explicaciones sobre la organización del calendario universitario en cuatrimestres de clases separados por períodos de exámenes, se menciona la necesidad de seguir un programa respetando los plazos o de preparar material pedagógico, tales como esquemas en transparencias. ${ }^{60} \mathrm{Se}$ señala además que dar clases implica contestar a las preguntas de los estudiantes, ${ }^{61}$ las cuales permiten introducir en el libro terminología y contenido científico. Michel Petit indica que muchas veces explicaciones teóricas o técnicas relativas a la profesión representada se incluyen en los thrillers especializados a través de la narración o de los diálogos entre profesionales, ${ }^{62}$ lo que según Jean Pierre Charpy tiene como objetivo crear realismo y aportar verosimilitud científica a la situación descrita, a pesar de que, para favorecer la comprensión del lector, sea necesario añadir explicaciones que, de hecho, no se harían en la realidad pues forman parte del saber de los profesionales..$^{63} \mathrm{En}$ La Cátedra, al corresponder a las interrogaciones de los estudiantes, estas explicaciones de tipo científico no resultan inverosímiles. No son aclaraciones artificialmente incluidas en el relato e inexistentes en lo real, pues no se dirigen a personajes especialistas en la materia, los cuales no necesitarían tales esclarecimientos, sino a personas que por su situación requieren estas informaciones. Así, la novela presenta varias veces diálogos entre una estudiante y su profesor que contienen términos y datos especializados:

-(...) Cuando utiliza la ecuación de Maxwell no veo en qué parte tiene en cuenta que la onda electromagnética atraviesa dos medios distintos.

-Claro que lo he tenido en cuenta. Por eso hemos utilizado dos valores del índice de refracción. (...)

-Y también está el problema del coeficiente de reflexión... -¿Qué problema del coeficiente de reflexión?

-Es que no he visto que, cuando la onda penetra en el segundo medio, se tenga en cuenta que la densidad de energía disminuya al llegar al segundo medio por la reflexión ${ }^{64}$

La función de este pasaje no radica en hacer que el lector entienda de qué se trata, pues las explicaciones dadas son demasiado complejas para alguien que desconoce el tema evocado. Tiende más bien a representar de manera realista una escena típica de la vida profesional del docente.

Pero las actividades diarias no sólo consisten en dar clase, sino también en realizar tareas administrativas, que van desde la firma de documentos burocráticos o de facturas hasta la convocación de reuniones para la reforma de los planes de estudio o problemas en materia de recursos humanos. ${ }^{65}$ Sin embargo, la mayor parte de su trabajo reside en la 
investigación. Se nos explica que un universitario es también un investigador, trabaja en un laboratorio, ya existente o creado por sí mismo. ${ }^{66} \mathrm{El}$ director de investigación orienta a los doctorandos, en especial a los que realizan parte de sus búsquedas en universidades extranjeras, participa en comisiones y tribunales de tesis doctorales, también acoge a jóvenes científicos recién diplomados para realizar estudios postdoctorales. ${ }^{67}$ Aparte del trabajo en el laboratorio, el universitario difunde el resultado de sus investigaciones a través de publicaciones y de comunicaciones en congresos. Se precisa que un artículo es redactado individualmente o en equipo, y que, después de la fase de redacción, se propone a revistas especializadas incluso extranjeras, que pueden rechazarlo, aceptarlo o pedir que sea modificado, lo cual implica un trabajo suplementario de corrección. Las publicaciones permiten evaluar los resultados de la investigación del universitario y posiblemente proporcionarle el reconocimiento de la comunidad científica internacional. ${ }^{68} \mathrm{La}$ participación en congresos, que pueden tener lugar en países extranjeros como Estados Unidos o Italia, ${ }^{69}$ implica enviar previamente un resumen de la comunicación dentro de un plazo determinado al comité científico responsable de la selección de las presentaciones, y luego redactar una comunicación que se presenta oralmente. ${ }^{70}$ También supone tareas administrativas, en especial escribir un informe sobre el congreso en que se participó. ${ }^{71}$ Del mismo modo que las cuestiones de los estudiantes permiten introducir en el relato contenido científico, el diálogo entre los candidatos a un puesto de universitario y los miembros del tribunal posibilita la inserción de precisiones sobre sus actividades de investigador sin que esas explicaciones parezcan inverosímiles. La entrevista de uno de los tres candidatos a la cátedra proporciona al lector detalles sobre el desarrollo del trabajo en un laboratorio y la realización de publicaciones:

-(...) Durante su estancia en Hamburgo participó en un proyecto sobre radiaciones de pulso corto. Sin embargo, cuando veo la relación de sus trabajos sólo encuentro una publicación sobre este tipo de radiación. ¿Es eso todo lo que ha sacado usted en limpio después de un año en Alemania? Me parece un bagaje bastante modesto. ¿Puede comentar eso?

-Sí (...). En primer lugar quiero señalar que cuando alguien llega a un laboratorio extranjero se necesita un cierto tiempo de preparación para el trabajo. En el caso de un proyecto experimental, como en el que yo participaba, se necesita adquirir el conocimiento de la instrumentación nueva, realizar modificaciones y calibraciones en dicha instrumentación, en fin, poner las cosas a punto. Sólo después de eso comienza realmente el trabajo que puede dar lugar a publicaciones científicas. Este hecho quizá pueda parecer extraño al que nunca ha pasado por esta experiencia, pero puedo asegurarle que es algo bien conocido por los que hemos tenido la inquietud de completar nuestra formación científica fuera de España. ${ }^{72}$

La referencia al trabajo de investigación en laboratorios extranjeros permite además aludir a las condiciones de trabajo de los universitarios españoles y compararlas en particular con las condiciones laborales de los investigadores norteamericanos. En Estados Unidos, se paga mucho más a los investigadores, los laboratorios no tienen nada que ver y los presupuestos son cinco o diez veces superiores. ${ }^{73}$

Por fin, las actividades de un universitario también consisten en juzgar a sus iguales. Participan en su contratación actuando en tribunales de oposición. Sin embargo, el tema de las oposiciones no sólo permite al lector familiarizarse con este aspecto del mundo académico, sino que se presenta como ingrediente esencial del thriller pues desempeña un papel importante en el argumento del libro: de su presencia surge el suspense de la novela. Por tanto, este tema no sólo permite informar al lector sobre el sector profesional donde sucede la historia. De él depende la progresión del argumento, es imprescindible 
para el desarrollo de la narración, con lo cual es un aspecto propio de ese sector laboral y, a la vez, un elemento de ese medio profesional indispensable para la existencia del thriller.

\section{Realidades profesionales componentes del thriller académico}

Como lo señalamos anteriormente, para Michel Petit los thrillers especializados se definen por una representación de un mundo profesional que se realiza dentro de un marco genérico específico, el thriller, para llamar la atención del público de masa. ${ }^{74}$ Pero más que un thriller o una representación de un sector laboral, La Cátedra es un thriller especializado, o sea un thriller fuertemente relacionado con un medio profesional que a su vez constituye un thriller: el tema principal de la novela, las oposiciones, corresponde a una faceta del mundo académico que se convierte en componente fundamental del thriller.

Los tribunales de oposición para obtener un puesto de catedrático constituyen el motivo central de la obra, tal y como lo indica el título, que se refiere precisamente a una oposición para obtener una cátedra. Su representación permite que el lector tenga información sobre este aspecto del sector universitario. Así, varias explicaciones hacen que nos familiaricemos con la organización y el funcionamiento de las oposiciones. Aparece primero una publicación oficial en el Boletín del Estado, ${ }^{75}$ luego se forma una comisión compuesta de diferentes catedráticos especializados en el campo correspondiente a la cátedra, procedentes de diversas universidades y la mayoría elegidos por sorteo. ${ }^{76} \mathrm{La}$ duración de las oposiciones es de varios días, pues los miembros del tribunal deben examinar las candidaturas, escuchar a los candidatos y redactar informes. ${ }^{77}$ Todos pueden asistir a las entrevistas de los candidatos ya que son sesiones públicas pero hay poca gente que viene, a no ser los familiares de los candidatos, su pareja, profesores o doctorandos de la universidad. ${ }^{78}$ Cada candidato tiene que presentar oralmente su historial académico y profesional, explicar sus investigaciones, sus programas y métodos de docencia, certificar sus actividades entregando al tribunal documentos como memorias o proyectando gráficos a partir del ordenador. ${ }^{79}$ Después tienen que contestar a las preguntas del tribunal..$^{80} \mathrm{De}$ la misma manera que los diálogos entre estudiantes y docentes o entre candidatos y miembros de la comisión permiten insertar en el texto explicaciones teóricas o técnicas, las explicaciones solicitadas por ciertos personajes hacen posible clarificar el desarrollo de las oposiciones. ${ }^{81}$ Así, la representación del funcionamiento de las oposiciones facilita datos al lector sobre este aspecto del universo académico, pero más adelante vamos a ver que se erige en ingrediente policíaco de la novela.

Un lector que desconoce el mundo universitario puede suponer que los procesos de contratación y la organización de tribunales de oposición se basan en el deseo de proponer el puesto de catedrático a una persona que demuestre tener las competencias requeridas. Sin embargo, la novela le enseña que otros criterios, no oficiales, llegan a ser más importantes a la hora de elegir un candidato: entran en juego intereses financieros vinculados con una especulación inmobiliaria de varios millones de euros. ${ }^{82}$ Desde el principio nos damos cuenta de que la realización de entrevistas y la convocación de una comisión son una mascarada pues el presidente del tribunal de oposición ya ha decidido quién obtendrá el puesto, aunque el candidato elegido para la cátedra no presente las competencias pedidas..$^{83} \mathrm{El}$ presidente no quiere que la opinión posiblemente divergente de ciertos miembros de la comisión pueda comprometer su decisión y trata de influir en su juicio poniéndose en contacto con ellos o con colegas que trabajan con ellos para 
obtener su apoyo, hacer que recomienden a uno de los candidatos, práctica que al final resulta corriente..$^{84}$ También intenta influirlos simpatizando con ellos, alabándolos, o invitándolos a tomar una copa ${ }^{85}$ Además, procura prescindir de los servicios de uno de ellos proponiendo una fecha que no le conviene o mintiéndole sobre el número de candidatos en la oposición para que no considere necesario desplazarse, para que renuncie, y permita así que lo reemplace un suplente amigo del presidente del tribunal. ${ }^{86}$ $\mathrm{E}$, incluso, quiere deshacerse de él e impedir que participe en el tribunal, mediante una denuncia falsa ${ }^{87}$ que se refiere a un delito relacionado con el mundo universitario, o sea al posible acoso sexual realizado por un profesor sobre una estudiante, que luego será asesinada ${ }^{88}$ pero que no se presenta como elemento principal sino secundario del thriller. Se evoca el presunto delito cometido y también se hace referencia al castigo previsto por las instancias universitarias en semejante caso pues, además de la sanción penal, el reglamento interno de la universidad incluye la eventualidad de que existan faltas disciplinarias y prevé investigaciones y sanciones internas; ${ }^{89}$ también se hace referencia a la existencia de denuncias falsas, abusivas. ${ }^{90}$ Los temas del acoso sexual y de las denuncias mentirosas pueden considerarse como aspectos del mundo académico, pero no son meros elementos realistas puestos al servicio de la representación de este medio sino ingredientes policíacos que forman parte integrante del thriller que constituye la novela. Son temas específicos del thriller académico, pero aquí no son los ingredientes centrales del argumento sino aspectos que se relacionan con el tema mayor del thriller académico: la oposición. Además, el Presidente de la Comisión trata de intimidar a los demás candidatos para que no se presenten. ${ }^{91}$ Amenaza a una candidata con represalias ${ }^{92}$ y si, a pesar de todo, decide presentarse, intentará deshacerse de ella desestabilizándola durante las entrevistas haciéndole preguntas para menospreciar su trabajo de investigación, de docencia y su experiencia en el extranjero. ${ }^{93}$ La referencia a otras oposiciones permite entender que las intimidaciones y la agresividad son aspectos corrientes en esas situaciones, pues son el escenario de ajustes de cuentas no siempre vinculadas con la oposición en sí misma o con el candidato elegido, sino con intereses personales o con problemas anteriores entre miembros del tribunal. ${ }^{94}$ Así, para impedir que la candidata se presente o hacer que se ponga en un estado que altere su presentación o imposibilite que la haga, tratan de infundirle miedo poniendo una cabeza de gato en su coche, también intentan matar a otro candidato en un accidente y secuestran a la estudiante con que un miembro del tribunal tiene una relación amorosa. ${ }^{95}$ Sin embargo, con esta serie de acontecimientos nos alejamos de la representación de la realidad para acercarnos a la ficción.

Aunque según Michel Petit un thriller especializado se caracteriza antes que nada por la representación de un mundo profesional, notamos por la presencia de estos elementos poco realistas que un thriller académico como La Cátedra se define ante todo por el hecho de ser un thriller, una ficción, que representa y denuncia ciertos aspectos del mundo universitario. Javier Piqueras subraya que para él se trataba de crear una buena ficción mezclando «misterio y acción con algo de humor y sexo, todo ello incrustado en el ambiente universitario $" .{ }^{96} \mathrm{Su}$ objetivo era crear una novela de intriga que representara personas y situaciones típicas del mundo académico para llamar la atención de los lectores que fueran universitarios, público que Jean-Louis Trouillon llama "lectores expertos » 0 " profesionales del sector tratado », ${ }^{97} \mathrm{y}$ también para informar a « la gente que no conoce el mundo universitario y que muchas veces tiene una idea superficial de la actividad universitaria, ${ }^{98}$ es decir el " gran público ${ }^{99}$ La dimensión realista de su novela no se puede negar puesto que, en cierto modo, Javier Piqueras es el portavoz de su medio 
laboral: nos señala que a sus colegas « les ha divertido reconocer ciertas situaciones que surgen, por ejemplo, en una oposición (...), o alguna característica de alguien conocido », ${ }^{100} \mathrm{e}$, incluso, le sugirieron « la inclusión de determinados problemas, actitudes o personas de la Universidad en el argumento de (sus) próximas novelas $» .{ }^{101}$ Sin embargo, descarta que su meta principal haya sido denunciar las facetas de su medio laboral:

En mis novelas hay algunas críticas a determinados aspectos del mundo universitario que son inevitables porque me propuse desarrollar la trama de intriga dentro de un marco, de un ambiente, realista. Creo que si el principal objetivo hubiera sido criticar aspectos universitarios, las novelas tendrían menos interés para el público general. ${ }^{102}$

Con todo, el argumento no se desarrolla dentro del marco narrativo de la novela realista, sino del thriller, más específicamente del thriller académico. Más allá de la representación del medio universitario, el substrato profesional nutre la trama del thriller, tal y como lo sugiere el peritexto. Por consiguiente, La Cátedra no es una novela realista o un campus novel sino un thriller académico: la intriga se funda en la denuncia de elementos irregulares, delictivos o criminales pertenecientes al mundo académico, y, sin ellos, no existiría, sería otra novela.

\section{BIBLIOGRAFÍA}

CHARPY, Jean-Pierre, « Le roman à suspense à dominante médicale : typologie d'un sous-groupe générique ", dans Aspects de la Fiction à Substrat Professionnel, université Victor Segalen Bordeaux 2, septembre 2004, p. 63-80.

ISANI, Shaeda, «The FASP and the Genres within the Genre », dans Aspects de la Fiction à Substrat Professionnel, université Victor Segalen Bordeaux 2, septembre 2004, p. 25-36.

PETIT, Michel, « La fiction à substrat professionnel : une autre voie d'accès à l'anglais de spécialité », Asp, no 23/26, 1999, p. 57-81.

-, «Quelques réflexions sur la fiction à substrat professionnel : du géneral au particulier », dans Aspects de la Fiction à Substrat Professionnel, université Victor Segalen Bordeaux 2, septembre 2004, p. 3-23.

Petit, Cécile, « Correo electrónico del 19 de febrero de 2009 de Javier Piqueras », sin editar.

-, «Preguntas a Javier Piqueras », 14 de enero de 2008, sin editar.

Piqueras De NoRiega, Javier, La Cátedra, Barcelona, Editorial Meteora, 2003, 382 p.

-, El Congreso, Barcelona, Editorial Meteora, 2005, 300 p.

TROUILLON, Jean-Louis, « La FASP et la motivation du lecteur : l'exemple du monde universitaire de David Lodge ", dans Aspects de la Fiction à Substrat Professionnel, université Victor Segalen Bordeaux 2, septembre 2004, p. 53-62. 


\section{NOTAS}

1. Javier Piqueras de Noriega, La Cátedra, Barcelona, Editorial Meteora, 2003, $382 \mathrm{p}$.

2. Javier PiQueras de Noriega, El Congreso, Barcelona, Editorial Meteora, 2005, 300 p.

3. Michel PETIT, «La fiction à substrat professionnel: une autre voie d'accès à l'anglais de spécialité », Asp, no 23/26, 1999, p. 57-81; Michel PETIT, "Quelques réflexions sur la fiction à substrat professionnel: du géneral au particulier», dans Aspects de la Fiction à Substrat Professionnel, université Victor Segalen Bordeaux 2, septembre 2004, p. 3-23.

4. Michel Petit, op. cit., 2004, p. 5.

5. Ibid., p. 6; Michel PeTIT, op. cit., 1999, p. 57-58.

6. "Also known as 'the campus novel', the academic novel is fiction situated in a university milieu which of course provides the substrat professionnel around which the plot pivots. Principally represented by Anglo-Saxon writers (Kingsley Amis, David Lodge, J M. Coetzee, Philip Roth, Tom Sharpe, etc...) » Shaeda ISANI, "The FASP and the Genres within the Genre », dans Aspects de la Fiction à Substrat Professionnel, Bordeaux: Université Victor Segalen Bordeaux II, septembre 2004, p. 32.

7. Javier Piqueras nos indica sus influencias: «Creo que el estilo de mis novelas tiene influencia cinematográfica o más bien de las series de televisión, en el sentido de que en un capítulo hay unas escenas que se dejan sin terminar y sobre las que se vuelve más adelante. Por otra parte, hay un tipo de películas en las que el protagonista se ve envuelto en problemas a los que, en principio es ajeno, y no entiende por qué están pasando ciertas cosas a su alrededor. (...) En cuanto a novelas, tengo que mencionar Der Kampus (...) del profesor de la Universidad de Hamburgo Dietrich Schwanitz. La influencia de esta novela no se debe a la trama, que tiene poco que ver con la trama de mis novelas, sino a la manera en que Schwanitz utiliza su gran conocimiento de la universidad para ambientar su novela y mostrar, y con frecuencia satirizar, algunos aspectos poco conocidos de la universidad y los universitarios. (...) Me gustan las novelas de intriga, misterio, thriller, ... Entre éstas me gustan las que constituyen una serie con el mismo protagonista y en las que no hay mucha sangre ni muchos cadáveres. Me gustan también los «legal thrillers» de los que hay varios buenos autores norteamericanos. En cine prefiero temas realistas de la vida corriente más que argumentos fantásticos o exagerados, y también, claro, películas policíacas y de juicios». Javier PIQUERAs dans Cécile P ETIT, «Preguntas a Javier Piqueras », 14 de enero de 2008, sin editar.

8. Jean-Pierre CHARPY, «Le roman à suspense à dominante médicale : typologie d'un sous-groupe générique ", dans Aspects de la Fiction à Substrat Professionnel, université Victor Segalen Bordeaux 2, septembre 2004, p. 67-68.

9. Ibid., p. 69.

10. Esta foto se refiere a un pasaje del libro: «Cuando entró, María estaba desnuda en la cama tendida de espaldas con un manojo de cintas de seda roja en la mano. El hombre la cogió sin decir nada y ató las muñecas de la chica a dos barrotes en la cabecera de la cama ». Javier PIQUERAS DE N ORIEGA, op. cit., p. 199.

11. Ibid., p. 5.

12. Jean-Pierre CHARPY, op. cit., p. 67-68.

13. Javier PIQUERAS DE NORIEGa, ibid., p. 5.

14. Michel Petit, op. cit., 1999, p. 60, 63; Michel Petit, op. cit., 2004, p. 6-7.

15. Jean-Pierre CHARPY, op. cit., p. 67-68, Michel PETIT, op. cit., 1999, p. 192.

16. Jean-Pierre CHARPY, ibid., p. 70. 
17. «Cette appartenance au milieu, ou (...) ces liens avec le milieu (...) qu'ils soient initialement attestés ou ultérieurement (re)construits, (...) sont en tout cas toujours explicitement revendiqués par le paratexte et constituent, à l'évidence, un argument important de la reconnaissance générique pour le lecteur ", Michel PETIT, op. cit., p. 6-7.

18. [En línea], <http./www.editorialmeteora.com/cast/llibre.php ?id_LLIBRE=10>, consultado en febrero de 2009.

19. De este modo nos enteramos de que Javier Piqueras da clases de Física del Estado Sólido y de Técnicas de Microscopía. Los términos claves con los que define sus ejes de investigación son: «caracterización de materiales, microscopia, defectos en cristales, semiconductores, luminiscenci, semiconductores nanocristalinos, nanohilos ». Especifica que es autor o coautor de unos 260 artículos científicos, y que en los últimos cinco años fue organizador o miembro de los comités científicos de diez congresos internacionales. Incluye en su página un vínculo que remite a la lista de las publicaciones recientes de su grupo de investigación. [En línea], <http.// material.fis.ucm.es/ piqueras/>, consultado en febrero de 2009.

20. Cécile PETIT, « Correo electrónico del 19 de febrero de 2009 de Javier Piqueras », sin editar.

21. Javier PIQUeRAS De NoRIEGa, ibid., p. 6.

22. Michel PETIT, op. cit., 1999, p. 58 (nuestra traducción).

23. Ibid.

24. Ibid., p. 196.

25. Javier PiQUeRAS DE NoRIEgA, op. cit., p. 20, p. 48-49.

26. Ibid., p. 29.

27. Ibid.

28. Ibid., p. 20, 29, 30, 108.

29. Ibid., p. 9-10, 29, 48.

30. Ibid., p. 9, 30.

31. Ibid., p. 9, 16, 30, 57.

32. Ibid., p. 48-51.

33. Ibid., p. 10, 16, 20, 30, 41, 49, 187-190, 211, 323.

34. Ibid., p. 30, 33, 34.

35. Ibid., p. 9, 17, 169-170.

36. Ibid., p. 59.

37. Ibid., p. 17, 42.

38. Ibid., p. 15, 42.

39. Ibid., p. 22.

40. Ibid., p. 10, 24, 25, 164.

41. Ibid., p. 32.

42. Ibid., p. 44.

43. Ibid., p. 49.

44. Ibid., p. 44.

45. Ibid., p. 323.

46. Ibid., p. 19, 25, 31, 41, 170.

47. Ibid., p. 10, 17-18, 30, 31.

48. Ibid., p. 326.

49. Ibid., p. 22, 213.

50. «Iba casi siempre vestido con un traje bastante arrugado también de color gris. El nudo de su corbata, siempre la misma, rara vez estaba razonablemente centrado y las camisas blancas que vestía parecían tener veinte años de uso.» Ibid., p. 22. "Nuria tenía casi siempre una expresión seria, acentuada por las anticuadas gafas y una manera sosa de vestirse basada en rebecas y faldas de tonalidades oscuras. »Ibid., p. 51. 
51. Ibid., p. 30.

52. Ibid., p. 49-50.

53. «El profesor Cañamero me conoce desde hace muchos años, desde que yo estudiaba formación profesional. Conocía a mi padre del pueblo donde pasábamos las vacaciones y a veces cazaban juntos. Luego me ha echado una mano algunas veces y también con este contrato que tengo ahora. » Ibid., p. 326; «El tal centro le parecía una manera apenas disimulada por la que algunos profesores con las conexiones oportunas pasaban a ser privilegiados crónicos y obtenían todo tipo de medios para su investigación. » Ibid., p. 18; « El profesor Casals se las había arreglado pocos años antes de jubilarse para conseguir que Nuria obtuviera una cátedra y premiar así la lealtad de su colaboradora, manifestada en cosas tan variadas como sustituirle en todas las clases que hiciera falta, preparar el café de media mañana o recordar al resto del personal el día de su santo. Nuria dio por supuesto que ella, como antigua mano derecha del maestro, sería su sucesora y se le reconocería una autoridad moral en el departamento. » Ibid., p. 49.

54. «Izquierdo era el profesor de la Facultad más aborrecido por los alumnos. Reunía todas las condiciones para ello: explicaba fatal, era antipático y suspendía más que nadie. » Ibid., p. 92 ; « Hizo una tesina en el departamento de Física Nuclear. Y estaba bastante cabreado porque el director era el famoso Saturnino, que no tenía ni puta idea de nada. Pero después se especializó en nuevas energías, e hizo una tesis doctoral impresionante. ", ibid., p. 26-27.

55. Ibid., p. 121, 296.

56. Ibid., p. 121. "Si Cañamero se entera, me puedo despedir de ser doctor alguna vez. » Ibid., p. 296.

57. Michel PetIT, op. cit., 1999, p. 69-70.

58. «Salvatierra soltó una expresión de contrariedad: ¡Joder! (...) La exclamación de disgusto de Luis se debía a que la participación en un tribunal suponía emplear varios días en escuchar las exposiciones, deliberar con los compañeros, redactar informes, etc. » Javier PIQUERAS DE NORIEGA, op. cit., p. 12. Ibid., p. 12-13, 51, 91, 236.

59. Ibid., p. 16, p. 18.

60. Ibid., p. 62 , p. 18, p. 72 .

61. Ibid., p. 18-20, p. 62.

62. Michel Petit, op. cit., 2004, p. 17-18, Michel Petit, op. cit., 1999, p. 70-71.

63. Jean-Pierre CHARPY, op. cit., p. 74-75.

64. Javier PIQUeRAS DE NORIEGA, op. cit., p. 19-20. «- Para no olvidarme, he hecho una lista de preguntas (...) / - Se trataba de la onda electromagnética que atraviesa dos medios y de los problemas matemáticos que hay para describir lo que pasa en la superficie de separación. Yo no he entendido lo del coeficiente de reflexión. / - (...) Lo que pasa es que la fracción de energía incidente reflejada en la superficie de separación se supone pequeña. / - Sí. Pero luego habló de disipación en la superficie... / - Pero es otro fenómeno distinto. Esa disipación es un problema de tipo técnico relacionado con la manera material en que acoplamos los dos medios. No entré en detalles que no hacen falta en este nivel y me limité a englobar todo eso en el coeficiente de disipación, que ha llamado sigma. / - ¡Ah! Ya veo. No me había enterado de lo que es la sigma. El cálculo en donde aparece por primera vez no lo entendí. No sé si tomé bien los apuntes. / Cristina tenía un gran cuaderno, que evidentemente era su cuaderno de apuntes de clase, abierto por una página en la que señalaba unas fórmulas con el dedo. (...) / - Me refiero a esta demostración. / - Sí. (...) Lo que tiene aquí es la fórmula de la densidad de energía incidente (...), que luego la transformamos cuando la radiación penetra en el segundo material... / - Una cosa que no entiendo (...) es si se refiere a densidad de energía en superficie o en volumen. / - En superficie. La fórmula corresponde a unidad de superficie. (...) / - ¿Ves? Al penetrar en el segundo material tenemos en cuenta la conservación de la energía. Aquí en esta fórmula (...) es donde añadimos el término que contiene sigma como factor de disipación. (...) / -Lo mejor es que lo veamos allí en la pizarra, así podemos escribir más cómodamente. / Señaló una pequeña pizarra de plástico blanco 
colgada en una pared del amplio despacho y que estaba llena de fórmulas y gráficos hechos con rotuladores de colores. Fueron hasta la pizarra dejando el cuaderno de apuntes en una mesa auxiliar que había a un lado. Después de borrar lo que había en la pizarra, Salvatierra cogió un rotulador y empezó a escribir. / - Bueno. Lo que estábamos aplicando era el principio de conservación de la energía - dijo mientras escribía unas fórmulas. / Cristina (...) se acercó para señalar un símbolo / - ¿Esto qué representa? / - Es la constante de atenuación de segundo medio.» Ibid., p. 56-59.

65. Ibid., p. 9, 10, 38, 63-64.

66. Ibid., p. 39.

67. Ibid., p. 10, 15, 22.

68. Ibid., p. 56.

69. Ibid., p. 10, 15.

70. Ibid., p. 35, 37, 164.

71. Ibid., p. 16.

72. Ibid., p. 243-244.

73. Ibid., p. 110. « En una de sus contestaciones proyectó una tabla con las cantidades que había recibido del Gobierno americano y de varias empresas americanas de alta tecnología para subvencionar su investigación. Las cifras levantaron un murmullo entre los asistentes, ya que eran cinco o diez veces superiores a las que podría obtener cualquier grupo destacado de investigación en España. »Ibid., p. 232.

74. Michel Petit, op. cit., 1999, p. 59.

75. Javier PIQUeRAS DE NORIEGA, op. cit., p. 11.

76. Ibid., p. 11-12.

77. Ibid., p. 12.

78. Ibid., p. 212, 231.

79. Ibid., p. 200, 206, 232, 234-235, 243.

80. Ibid., p. 232, 234-235, 243.

81. Ibid., p. 53.

82. Ibid., p. 69-71, 112 y siguientes, 264, 267, 278, 292, 299, 317-318.

83. «Esa cátedra está más que preparada para un amigo del jefe. El y Rosalía está en el tribunal. Parece que la cátedra está pensada para un tal Guzmán » Ibid., p. 42-43. Ibid., p. 66.

84. Ibid., p. 39, 72, 104-105, 112, 201, 271-273.

85. Ibid., p. 240-241.

86. Ibid., p. 34-35, 66, 73.

87. Ibid., p. 184-185, 241, 299, 303, 316.

88. Ibid., p. 126.

89. Ibid., p. 124-125, 149, 157, 184.

90. Ibid., p. 127.

91. Ibid., p. 77.

92. Ibid., p. 43, 46, 81. « Sí, Ana María tiene buen currículum. No sería bueno que opositara frente a Guzmán. Yo creía que no se iba a atrever. Desde luego, si se presenta se va a arrepentir. Eso lo tiene claro, se puede olvidar de sacar una cátedra aquí en su vida. » Ibid., p. 77. «- Si te presentas a esta oposición, no cuentes con ningún apoyo en el futuro. / - ¿Ahora tocan las amenazas? / - Ni amenazas ni nada. Si no cooperas cuando surge una cosa que es de interés general para la universidad, no cuentes con que te ayudemos más adelante. Olvídate de sacar ni esta cátedra ni ninguna otra. Y por supuesto te garantizo que, cuando se haga el Centro, no lo vas a pisar. ¿Está claro? Más te vale que pienses lo que haces. » Ibid., p. 80.

93. Ibid., p. 105, 233, 243-246.

94. Ibid., p. 53-55.

95. Ibid., p. 247, 271-273, 286-287, 317. 
96. Javier PIQUERAS, dans Cécile PETIT, op. cit.

97. Jean-Louis T RouILlon, «La FASP et la motivation du lecteur: l'exemple du monde universitaire de David Lodge », dans Aspects de la Fiction à Substrat Professionnel, université Victor Segalen Bordeaux 2, septembre 2004, p. 61.

98. Javier PIQUERAS, dans Cécile PETIT, op. cit. Javier Piqueras habla del « desconocimiento general que hay en España sobre la Universidad y lo que se hace en ella. » Ibid.

99. Jean-Louis Troulllon, op. cit., p. 61.

100. Javier PIQUERAS, dans Cécile PETIT, op. cit.

101. Ibid.

102. Ibid.

\section{RESÚMENES}

Javier Piqueras de Noriega, universitario y escritor español, publicó en 2003 su primera novela, titulada La Cátedra. Este libro inaugura en España el nacimiento de un género hasta ahora descartado por los autores españoles, y con todo frecuentemente desarrollado por autores anglófonos, o sea la FASP, género definido por el anglicista francés Michel Petit, y, en particular, la FASP académica. El estudio de las características externas e internas de La Cátedra muestra que esta novela es un excelente thriller universitario. La Cátedra no se presenta como una simple representación realista del mundo de la universidad que se haría en el marco de un género elegido para atraer al público de masa, sino como un thriller cuya existencia depende, precisamente, del substrato profesional representado y denunciado.

Javier Piqueras de Noriega, universitaire et écrivain espagnol, a publié en 2003 un premier roman intitulé La Cátedra. Ce livre inaugure en Espagne la naissance d'un genre jusqu'a présent boudé par les écrivains espagnols et cependant bien connu des auteurs anglophones, à savoir la FASP, genre initialement défini par l'angliciste Michel Petit, et plus précisément la FASP universitaire. L'étude des caractéristiques internes et externes de La Cátedra montre qu'il s'agit d'un thriller universitaire très réussi. Ce roman ne se présente pas comme une simple représentation réaliste du monde de l'université qui se ferait dans le cadre d'un genre censé attirer le grand public, mais comme un thriller dont l'existence dépend justement du substrat professionnel représenté et dénoncé.

Javier Piqueras de Noriega, a scholar and Spanish writer, published his first novel entitled La Cátedra in 2003. This book marks the birth of a genre in Spain which was previously neglected by Spanish writers, however well-known in English-speaking circles. This genre is the FASP (thrillers embedded in a specialized or professional environment), which was initially defined by anglicist Michel Petit; here it is more specifically academic FASP. Studying the external and internal characteristics of La Cátedra shows that it is a well-achieved academic thriller. This novel does not present itself as simply a realistic representation of the academic world, which would take place within the framework of a genre aimed at a wider readership, but as a thriller whose existence depends precisely on this professional environment which is at once featured yet denounced. 
ÍNDICE

Palabras claves: Campus novel, Thriller académico, FASP, Literatura hispánica, Javier Piqueras de Noriega

Keywords: Campus novel, academic thriller, FASP, hispanic literature, Javier Piqueras de Noriega Mots-clés: Campus novel, Thriller universitaire, FASP, littérature hispanique, Javier Piqueras de Noriega

\section{AUTOR}

\section{CÉCILE PETIT}

Université Paris 3 Sorbonne Nouvelle (CRICCAL)

Université Evry Val d'Essonne 